\title{
A DOUBLY-INFINITE SYSTEM OF SIMPLE GROUPS.
}

\section{(Abstract of a Paper * presented to the Congress of Mathematics at Chicago, August 25, 1893.)}

BX PROF. E. HASTINGS MOORE.

1. List of orders of systems of simple groups.

The following is, so far as I know, a complete list of the orders of systems of simple groups which have as yet been determined ( $q=$ prime, $n=$ positive integer).

$$
q \text {. }
$$

$$
\begin{array}{ll}
\frac{1}{2} n !, & (n>4) . \\
\frac{1}{2} q\left(q^{2}-1\right), & (q>3) ;
\end{array}
$$

the group of the modular equation for the transformation of alliptic functions of order $q$.

$$
\begin{aligned}
\frac{1}{2} q^{n}\left(q^{2 n}-1\right), & (q>2,(q, n) \neq(3,1)) . \\
q^{n}\left(q^{2 n}-1\right), & (q=2, n>1) .
\end{aligned}
$$

This system of simple groups is a generalization of the system (3) to be explained in this paper.

$$
\frac{\left(q^{n}-1\right) q^{n-1}\left(q^{n-1}-1\right) q^{n-2} \ldots\left(q^{2}-1\right) q}{\delta}((q, n) \neq(2,2),(3,2)),
$$

in which $\delta=[n, q-1]$, the greatest common divisor of $*$ and $q-1$.

(5) $\frac{1}{2}\left(q^{2 n}-1\right) q^{2 n-1}\left(q^{2 n-2}-1\right) q^{2 n-3} \ldots\left(q^{2}-1\right) q, \quad(q>2)$.

$$
\left(q^{2 n}-1\right) q^{2 n-1}\left(q^{2 n-2}-1\right) q^{2 n-3} \ldots{ }^{\prime}\left(q^{2}-1\right) q, \quad(q=2, n>2) .
$$

(6) $\quad\left(P_{n}-1\right) 2^{2 n-2}\left(P_{n-1}-1\right) 2^{2 n-4} \ldots\left(P_{2}-1\right) 2^{2} . \quad(n>2)$.

in which $P_{n}=2^{2 n-1}+2^{n-1}$

* This paper will be publisbed in full in the Proceedings of the Con. gress. It will also appear as the first part of a paper, "The sub.groups of the simple group whose order is $\frac{1}{q n}\left(q^{2 n}-1\right)$ if $q>2$, or $q^{n}\left(q^{2 n}-1\right)$ If $s=2$," to be published in the Mathematieche Annalen. 
The systems* (4), (5), (6) are either given by Jordan or are derived from Jordan's decompositions of certain linear groups by the principle that the quotient-group $f$ of any two consecutive groups in the series of composition of any group is a simple group. $\ddagger$

The systems (1), (2), (3), (b) are simply infinite; the systems $\left(3^{\prime}\right),(4),(5)$ are doubly infinite. It is clear that of the three doubly-infinite systems the new system $\left(3^{\prime}\right)$ is the densest, that is, its orders increase least rapidly as $q$ and $n$ increase.

Professor Cole discovered last spring a new simple group, of order 504 not contilined in the six systems (1), (2), (3), (4), (5), (6). The facts (a) that in the system (3') the group having $(q, n)=(3,2)$, order 360 , had previously been identified as holoedrically isomorphic with the alternating group in six letters (a simple group), and (b) that the group having $(q, n)=(2,3)$ had the order 504 of Cole's new simple group, led to the present investigation.

The simple groups of composite order $<660$ have been completely enumerated by Hölder (Math. Annalen, vol. 40) and Cole (Amer. Journal of Math., vol. 14; Bulletin of New York Math. Socretr, vol. 2, p. 254, foot-note). They are one group each for the orders $60,168,360$ and 504 . These are all included in the new system $\left(3^{\prime}\right)$, heing the groups having, respectively, $(q, n)=(5,1)$ or $(2,:),(\tau, 1)$. $(3,2)$, and $(2,3)$.

\section{T'he simply-infinite system (3) of simple groups of wriler $\frac{1}{2} q\left(q^{2}-1\right), \quad(q>3)$.}

The formula

$$
\Leftrightarrow^{\prime} \equiv \frac{\alpha \omega+\beta}{\gamma \omega+\delta} \quad(\bmod . q)
$$

where

$$
\alpha \delta-\beta \gamma \equiv 1 \quad \text { (moll. } q)
$$

and where $\alpha, \beta, \gamma, \delta$ are integers taken modulo $q$, and (s). (a)' run throngh the series of $q+1$ vulues $0,1,2, \ldots \overline{-1}, \infty$, may be considered an analytic expression of a certain substiution on the $q+1$ symbols or marks $0,1,2, \ldots \overline{q-1}, \infty$. The totality of all such distiuct substitutions constitutes a

* See Jordan, “Traité des Substitutions,” (4) p. 106, (5) pp. 176, 178. (6) pp. 205, 218. These references were given by Professur Cole in the paper, "Ou simple groups," presented by him to the Congress.

+ See Holder, Math. Annalen, vol. 34.

$\ddagger$ Two systems of order $(6)$ are given. 
group of order $\frac{1}{2} q\left(q^{2}-1\right)$, a particular form of the (abstract) group in question. The group is for every $q>3$ simple. For un admirable exposition of the properties of this group, together with further references, see Klein-Fricke, "Modulfunctionen," vol. I, pp. 419-491.

The existence and properties of the abstract group as studied under this form depend above all things upon

(a) The existence of the system of $q$ marks, $0,1,2, \ldots$ $q-1$, which may he combined by the four fundamental operations of algehra, and in which the $q-1$ marks $(0$ exclnded) are given as the snccessive powers of one of them (a primitive congruence-root, modulo $q$ );

(b) The introduction of the mark $\infty$ (due to Galois).

\section{The Cialois-field of order $s=q^{n}$.}

Suppose we have a system of symbols or markis, $\mu_{1}$, $\mu_{2} \ldots \mu_{s}$, in number $s$, and suppose that these $s$ marks may be combined by the four fundamental operations of algebraaddition, snbtraction, multiplication, and division-the operations being subject to the ordinary abstract operalional laws of algebra $\left(\mu_{f}+\mu_{g}=\mu_{g}+\mu_{f}, \mu_{f} \mu_{g}=\mu_{g} \mu_{f}\right.$, etc. $)$, and that when the mirks are so combined the results of these operations are in every case uniquely deternined and belong to the system of marks. Such a system of $s$ marks we call $a$ field "if orders.

The niost fumiliar instance of such a field, of order $:=q=a$ prime, is the system of $q$ incongruous classes (modulo $q$ ) of rational integral numbers $a$.

Galois discoverel an important generalization of the preceding field. Let $F_{n}(\xi)=\sum_{0}^{n} c_{\iota} \xi_{\iota}$, where the $c_{\iota}$ are integers and $r_{n}=1$, be irreducible, modulo $q$. Then the Galois-field of order $s=q^{n}, G E\left[q^{n}\right]$, consists of the system of $q^{n}$ incongruous classses (modulis $q, F_{n}(\xi)$ ) of 1ational integral functions of $\xi$ with integral coefficients. In this $G F\left[q^{n}\right]$ there exist primitive ronts; the $q^{n}-1$ snccessive power's of a primitive root are the $q^{n}-1$ marks of the field (0 excluded). The $G F\left[q^{n}\right]$ is uniquely defined for every $q=$ prime, $n=$ positive integer ; that is,

(1) $F_{n}(\xi)$ which are irrelucible (modulo $q$ ) do exist;

(2) The $G F\left[q^{n}\right]$ is indepeudent of the particular $F_{n}(\xi)$ used in its construction.

For the detilils of this Galois theory, see serret, "Algèbre supérieure," 5th edition, vol. Ii pp. 122-189. and Jordan, 'Substitutions," pp. 1t-18.

It should be remarked further that every field of order $s$ is in fact abstractly considered a Galois-field of order $s=q^{n}$. 


\section{The doubly-infinite system (3') of simple* groups of order} $\frac{1}{2} q^{n}\left(q^{2 n}-1\right)$ if $q>2$, or $q^{n}\left(q^{2 n}-1\right)$ if $q=2$.

Let $\alpha, \beta, \gamma, \delta$ be marks of the $G F\left[q^{n}\right]$ satisfying the equation $\alpha \delta-\beta \gamma=1$. Let $\omega, \omega^{\prime}$ be variable marks, each assuming, besides the $q^{n}$ values of the $G F\left[q^{n}\right]$, the value $\infty$. The formula

$$
\omega^{\prime}=\frac{\alpha \omega+\beta}{\gamma \omega+\delta} \quad(\alpha \delta-\beta \gamma=1)
$$

expresses a certain substitution on the $q^{n}+1$ marks $\omega$. The totality of all such distinct substitutions constitutes a group of order $\frac{1}{2} q^{n}\left(q^{2 n}-1\right)$ if $q>2$ or $q^{\prime \prime}\left(q^{2 n}-1\right)$ if $q=2$, a particular form of the (abstract) group in question.

For brevity we write hereafter s for $q^{n}$ and $M(s)=M\left(q^{n}\right)$ for the order. I prove in this paper that this group $G_{\text {ML(o) }}$ is a simple group in all except the two particular cases

when the $G_{M(s)}$ are in fact known to be

$$
\left\{\begin{array}{l}
n=1, q=2, s=2, M(s)=6 \\
n=1, q=3, s=3, M(s)=12
\end{array}\right.
$$

the $G_{6}=3$ : symmetric substitution-group on $s+1=3$ letters,

the $G_{12}$ tetraedron group or alternating substitution-group on $s+1=4$ letters,

and to have as self-conjugate sub-groups

$$
\left\{\begin{array}{l}
\text { a } G_{2} \text { cyclic-group. } \\
\text { a } G_{4} \text { four-group. }
\end{array}\right.
$$

To this end it is necessary first to discuss the individual operators and the cyclic and commutative $f$ sub-groups of the $G_{M(s)}$, and secondly to establish a diophantine equation for the oriler of a self-conjugate sub-group, which shall lead to the conclusion that the only self-conjugate sub-groups of the $G_{M(s)}$ ar'e the identity and the $G_{M(s)}$ itself.

\section{The individual operators and the cyclic and commutative sub-groups of the $G_{\text {sas). }}$.}

By an investigation differing somewhat in details from that of Serret $\ddagger$ and Gierster $\&$ for the case $n=1$, as given

$*(q, n)=(2,1),(3,1)$ excepted.

+ A group is called commutative if its operntors are commutative.

¥ Sirret: Comptes Rendus, 1859, 1860 ; "Algebre supérieure," vol. rr, p. $8133 \mathrm{ff}$.

\& Gierster: Mnth. Annalen, vol. 18, pp. 819-865; “ Die Untergruppen der Galois'schis" Gruppe der Moilulurgleichungen fur den Fall eiue? primzabligeu Trunsformutionsg rades." 
in Klein-Fricke, "Modulfunctionen," vol. I, pp. 410-450, it is found that every substitution (the identity excepted) ilitermines and lies in one and only one largest commmntafive sub-group. These sub-groups may be arranged in three different sets, the groups of each set being conjugate with one unother under the $G_{M(s)}$.

I. $s+1$ conjugate commutative $G_{s=q^{n}}$. Theso $s^{2}-1$ sulstitutions are all of period $q$, and for $q=2$ they are all conjugate, while for $q>2$ they separute into two sets of $\frac{1}{s}\left(s^{2}-1\right)$ conjugate substitutions. "The $q-1$ substitutions of a cyclic group $G_{a}$ belong

for $q>2\left\{\begin{array}{l}n \text {. odd, half to one and half to the other } \\ n \text { even, all to the same }\end{array}\right\}$ set of conjugate substitutions.

If. $\frac{1}{2} \times(s+1)$ conjugate cyclic groups $G_{s-1}$ if $q>2$, or: $G_{s-1}$ if $q=2$.

III. $\frac{1}{2} s(s-1)$ conjugate cyclic groups $G_{\frac{a+}{2}}$ if $q>2$, or G.+1 if $q=2$.

\section{The diophantine equation for the order of a self-conjugate sub-group of the $G_{\text {a(s). }}$.}

Let $G_{d}$ be $n$ self-conjugate sub-group of the $G_{m(s)}$. If it contuins one of a set of conjugate substitutions or subgroups of the $G_{M(s)}$, it will contitin all of that set. Whence the $G_{d}$ contains $\frac{1}{2} *(s+1)$ conjugate $G_{d_{-}}$from the conjugate

groups (II), and $\alpha_{2} \times(*-1)$ conjugate $G_{a_{+}}$from the conjugate groups (III).

Further, the $T_{n}$ contains either no substitution of period $q$, or

$\left\{\begin{array}{l}q>2, n \text { odd, all of both sets } \\ q>2, n \text { even, all of one set or of both sets } \\ q=2, \text { all of the one set }\end{array}\right\}$ of conjugate $q=2$, all of the one set
substitutions of period $q$.

The enumeration of the substitutions of the self-conjugate $G_{d}$ leads to the diophantine equation

$$
-\frac{1}{2}\left(s^{2}-1\right) h+\frac{1}{2} s(s+1) d_{-}+\frac{1}{2} s(s-1) d_{+}=d,
$$

to be satisfied for positive integral values of $d, d_{-}, d_{+}, h$, where $d, d_{-}, d_{+}$are divisors of $\frac{1}{2} \times\left(s^{2}-1\right), \frac{1}{2}(s-1), \frac{1}{2}(s+1)$, respectively, if $q>2$, or of $s\left(s^{2}-1\right), s-1, s+1$, respectively, if $q=2$, and where $h$ has the value

$\{h=2$ or 0, for $q>2, n$ odd, and for $q=2$,

$\{h=2,1$ or 0 , for $q>2, n$ even. 
From this equation follows the simplicity of the $G_{M(s)}$ in all except the two pirticular cases $(q, n)=(2,1)$ and $(3,1)$.

The University of Chicago, October 18, 1893.

\section{NOTE ON MONOGENIC FUNCTIONS OF A SINGLE VARIABLE.}

By PIOF. T, CRAIG.

THE following remark is so obvious that it seems impossible that it has not been made before; still neither the writer nor those to whom he has spoken have seen it.

Suppose $P(x, y), Q(x, y)$ to be real functions of the real variables $x, y$. Form $P+i Q$ : in order that this shall be a monogenic function of $x+i y=z$ it is necessary first that $P$ and $Q$ be functions satisfying Iaplace's equation

$$
\begin{aligned}
& \nabla P=\frac{\partial^{2} P}{\partial x}+\frac{\partial^{2} P}{\partial y^{2}}=0, \\
& \nabla Q=\frac{\partial^{2} Q}{\partial x^{2}}+\frac{\partial^{2} Q}{\partial y^{2}}=0 .
\end{aligned}
$$

But it is not sufficient that $P$ and $Q$ satisfy this equation. If $P$ be a solution of the equation, $Q$ must be determined by aid of Cauchy's equations

(1)

$$
\frac{\partial P}{\partial x}-\frac{\partial Q}{\partial y}=0
$$

$$
\frac{\partial P}{\partial y}+\frac{\partial Q}{\partial x}=0
$$

that is, $Q$ will be given by the integral

$$
Q=\int_{\left(x_{0}, y_{0}\right)}^{(x, y)}-\frac{\partial P}{\partial y} d x+\frac{\partial P}{\partial x} d y,
$$

in which the condition of integrability is satisfied, since $\nabla P=0$. 